\title{
ON THE FUNDAMENTAL GROUPS OF COMPACT SASAKIAN MANIFOLDS
}

\author{
XiaOyAng Chen
}

\begin{abstract}
We study the fundamental groups of compact Sasakian manifolds, which we call Sasaki groups. It is shown that the fundamental group of any compact Hodge manifold is Sasaki. In particular, all finite groups are Sasaki. On the other hand, we show that there exist many restrictions on Sasaki groups. We also study the Abel-Jacobi map of a compact Sasakian manifold and its applications to Sasaki groups.
\end{abstract}

\section{Introduction}

Recently Sasakian manifolds have attracted a lot of attention; see [5] for a comprehensive treatment. However, very little is known for the fundamental groups of compact Sasakian manifolds. Here we make some steps in this direction.

Sasakian manifolds are odd-dimensional analogues of Kähler manifolds. Recall that a Riemannian manifold $\left(M^{2 n+1}, g\right)$ (we always consider connected manifolds in this paper) is Sasakian if it has a unit Killing vector field $\xi$, satisfying the equation

$$
R(X, \xi) Y=g\langle\xi, Y\rangle X-g\langle X, Y\rangle \xi
$$

Given such a characteristic vector field $\xi$ (also called Reeb vector field), we define a $(1,1)$ tensor $\phi$ by $\phi(X)=\nabla_{X} \xi$ and the characteristic one form $\eta$ by $\eta(X)=g\langle X, \xi\rangle$. Altogether we call $(g, \xi, \eta, \phi)$ a Sasakian structure. The vector field $\xi$ defines the characteristic foliation $F_{\xi}$ with one-dimensional leaves and the kernel of $\eta$ denoted by $D$, called the contact bundle, inherits an almost complex structure by restriction of $\phi$. Let $g^{T}=g-\eta \otimes \eta$. It turns out that $(g, \xi, \eta, \phi)$ is a Sasakian structure iff $\left(D, g^{T},\left.\phi\right|_{D}, d \eta\right)$ defines a transversal Kähler structure with transversal Kähler form $d \eta$.

A Sasakian structure on $M$ is called quasi regular if all leaves of the characteristic foliation $F_{\xi}$ are closed, otherwise it is called irregular. By a theorem of Wadsley [41], if a Sasakian structure on $M$ is quasi regular, $\xi$ generates a locally free $S^{1}$ action on $M$. A Sasakian structure on $M$ is called regular if this action is free. If the Sasakian structure on $M$ is quasi regular, then the quotient space $M / F_{\xi}$ is a Kähler orbifold. In general, there is no quotient space if it is irregular.

There is a natural transversal Levi-Civita connection $\nabla^{T}$ on $M$ defined by $\nabla_{X}^{T} Y=$ $\left[\nabla_{X} Y\right]^{p}$ if $X, Y \in D$ and $\nabla_{\xi}^{T} Y=[\xi, Y]^{p}, \nabla_{X}^{T} \xi=0$, where $Z^{p}$ denotes the projection of $Z$ to $D$ for any $Z \in T M$ and $\nabla$ is the usual Levi-Civita connection induced by $g$.

Received by the editors March 02, 2012. 
Now define the transversal curvature tensor $R^{T}$ by

$$
\begin{gathered}
R^{T}(X, Y) Z=\nabla_{X}^{T} \nabla_{Y}^{T} Z-\nabla_{Y}^{T} \nabla_{X}^{T} Z-\nabla_{[X, Y]}^{T} Z, \\
R^{T}(X, Y, Z, W)=g\left\langle R^{T}(X, Y) Z, W\right\rangle
\end{gathered}
$$

where $X, Y, Z, W \in D$.

We say that $(M, g)$ has nonpositive transversal sectional curvature if and only if $R^{T}(X, Y, Y, X) \leq 0$, for any two vectors $X, Y \in D$. Examples of compact Sasakian manifolds with nonpositive transversal sectional curvature are given by the BoothbyWang fibrations over compact Hodge orbifolds with nonpositive sectional curvature. See section 2 for details on the Boothby-Wang fibration.

We say that a finitely generated group is Sasaki (Kähler) if it is the the fundamental group of some compact Sasakian (Kähler) manifold.

It is known that any compact Sasakian manifold has even first Betti number; see $[4,19,39]$. Since one can lift a Sasakian structure to the covering space of a Sasakian manifold, the following proposition is immediate.

Proposition 1.1. A group that contains a finite index subgroup with odd first Betti number is not Sasaki. In particular, nontrivial-free groups cannot be Sasaki.

The next proposition gives many examples of Sasaki groups.

Proposition 1.2. Suppose $\Gamma$ is the fundamental group of some compact Hodge manifold, then $\Gamma$ is also a Sasaki group.

Using a classical result of Serre [1] that any finite group is the fundamental group of some compact Hodge manifold and also Propositions 1.1 and 1.2, one easily gets

Corollary 1.3. All finite groups are Sasaki and an abelian group is Sasaki if and only if it has even rank.

It was conjectured that any Kähler group is the fundamental group of some compact Hodge manifold. So it is natural to propose the following:

Conjecture All Kähler groups are Sasaki.

It is easy to see that there exist Sasaki groups which are not Kähler. For example, the discrete, torsion-free and cocompact subgroups of real Heisenberg group $H^{2 n+1}$ are Sasaki. However, they are not Kähler if $n \leq 3$ by a theorem of Carlson and Toledo; see $[10]$.

The next theorem tells us that some well-known results for Kähler groups remain true for Sasaki groups.

Theorem 1.4. Suppose $\Gamma$ is a Sasaki group, then

(1) $\Gamma$ has either zero or one end. In particular, $\Gamma$ cannot split as a nontrivial-free product.

(2) If $\Gamma$ is solvable, it contains a nilpotent subgroup of finite index.

The next theorem classifies those Sasaki groups that are also the fundamental group of some compact three-dimensional manifold.

Theorem 1.5. Suppose $\Gamma$ is a Sasaki group, then it is the fundamental group of some compact three-manifold $V^{3}$ if and only if $V^{3}$ has geometry modeled on $S^{3}$ or the three-dimensional Heisenberg group or $S(2, \mathbb{R})$. 
Theorem 1.6. Suppose $\left(M^{2 n+1}, g\right)$ is a compact Sasakian manifold with nonpositive transversal sectional curvature, then $\pi_{1}\left(M^{2 n+1}\right)$ is an infinite group that cannot be realized as the fundamental group of any compact Riemannian manifold with nonpositive sectional curvature.

As a consequence of Theorem 1.6, we recover the following fact that was previous proved by Eberlein's splitting theorem [16]: suppose $\Gamma=\pi_{1}\left(M^{3}\right)$, where $M^{3}$ is a compact three-manifold with geometry modeled on $S \widetilde{L(2, \mathbb{R})}$, then $\Gamma$ cannot be the fundamental group of any compact Riemannian manifold with nonpositive sectional curvature.

Theorem 1.6 suggests to study a new class of infinite groups, which we hope to cover in the future.

The organization of this paper is as follows. In Section 2 we review two constructions in Sasakian geometry and prove Proposition 1.2. Theorems 1.4 and 1.5 are proved in Section 3. In Section 4, we study harmonic maps from compact Sasakian manifolds and some applications. In Section 5, we prove Theorem 1.6. In Section 6, we study the Abel-Jacobi map of a compact Sasakian manifold and its applications to Sasaki groups.

\section{Constructions in Sasakian geometry and proof of Proposition 1.2}

We first recall two basic constructions in Sasakian geometry. For details, see [5]. For simplicity, we restrict ourselves to the class of regular Sasakian manifolds.

2.1. Boothby-Wang fibration. Suppose $\left(N^{2 n}, \omega\right)$ is a compact Hodge manifold with integral Kähler form $\omega$. By a theorem of Kobayashi [25], there exists a principal circle bundle $P$ over $N^{2 n}$ and a connection form $\eta$ on $P$ such that $d \eta=p^{*} \omega$, where $p$ is the projection map. Let $M^{2 n+1}$ be the total space of $P$. Now define a Riemanian metric $g$ on $M^{2 n+1}$ by $g=p^{*} h+\eta \otimes \eta$, where $h$ is the associated Kähler metric for $\omega$ on $N^{2 n}$. It is not hard to check that $\left(M^{2 n+1}, g\right)$ becomes a Sasakian manifold with transversal Kähler form $\omega$. We say that $\left(M^{2 n+1}, g\right)$ is the Boothby-Wang fibration over $\left(N^{2 n}, h\right)$. It is not hard to extend this construction to the case when $\left(N^{2 n}, \omega\right)$ is a compact Hodge orbifold.

From the construction of Sasakian structure on $M^{2 n+1}$, it is easy to see $\left(M^{2 n+1}, g\right)$ has nonpositive transversal sectional curvature if $\left(N^{2 n}, h\right)$ has nonpositive sectional curvature. For example, $\left(N^{2 n}, h\right)$ is a compact locally Hermitian symmetry space of noncompact type.

2.2. Join construction. Suppose $M_{1}$ and $M_{2}$ are two compact Sasakian manifolds over compact Hodge manifolds $\left(N_{1}, \omega_{1}\right)$ and $\left(N_{2}, \omega_{2}\right)$, respectively. Then $\left(N_{1} \times\right.$ $\left.N_{2}, \omega_{1}+\omega_{2}\right)$ is also a compact Hodge manifold. Let $M_{1} * M_{2}$ be the Sasakian manifold over $N_{1} \times N_{2}$ coming from Boothby-Wang fibration. We say that $M_{1} * M_{2}$ is the join of $M_{1}$ and $M_{2}$. It turns out $M_{1} * M_{2}$ is an $M_{2}$ bundle over $N_{1}$; see [5]. It is easy to see compact Sasakian manifolds with nonpositive transversal sectional curvature are closed under join construction.

Now we give the proof of Proposition 1.2.

Proof. Suppose $\Gamma=\pi_{1}(N)$, where $N$ is a compact Hodge manifold. Let $M$ be the Sasakian manifold over $N$ coming from Boothby-Wang fibration. Let $V=M * S^{3}$. 
Then $V$ is a compact Sasakian manifold and also an $S^{3}$ bundle over $N$. From the long exact sequence of homotopy groups, one gets $\pi_{1}(V) \simeq \Gamma$.

\section{Orbifold fundamental groups of compact Kähler orbifolds}

We refer the readers to $[5,13,40]$ for definitions of orbifolds and orbifold fundamental groups.

Recently there has been much interest in Kähler orbifold groups, namely, the orbifold fundamental groups of compact Kähler orbifolds. For example, see [7, 35] for the related work. A fundamental question on this topic is the following:

Does the restrictions for Kähler groups extend to Kähler orbifold groups?

Or we can ask the following much stronger question:

Suppose $\Gamma$ is a Kähler orbifold group. Is it true that $\Gamma$ contains a subgroup of finite index $\Gamma_{0}$ such that $\Gamma_{0}$ is a Kähler group?

The work of Campana gives some partial answers to the above questions. More precisely, we have the following two lemmas. See [6, 7] for proof.

Lemma 3.1. Suppose $G$ is the orbifold fundamental group of a compact Kähler orbifold, then

(1) $G$ has zero or one end.

(2) If $G$ is solvable, it contains a nilpotent subgroup of finite index.

Remark: The first part of lemma 3.1 is not explicitly stated in [7]; however, it follows from the arguments there and the corresponding result in the manifold case.

Lemma 3.2. Suppose $\Gamma$ is a Kähler orbifold group and also residually finite. Then $\Gamma$ contains a subgroup of finite index $\Gamma_{0}$ such that $\Gamma_{0}$ is a Kähler group.

Recall that a group is said to be residually finite if and only if the intersection of all its subgroups of finite index is trivial. We also recall some basic facts on the end of a finitely generated group. See [15] for more details. Suppose $X$ is a locally compact connected topological space. The set of ends of $X$, denoted by $E(X)$, is defined as the inverse limit:

$$
\lim _{K \subseteq X} \pi_{0}\left(K^{c}\right),
$$

where $K$ is a compact subset of $X, K^{c}$ is the complement of $K$ and $\pi_{0}\left(K^{c}\right)$ is the number of path-connected components of $K^{c}$. If $\Gamma$ is a finitely generated group, the space of ends $E(\Gamma)$ is defined as the set of ends of its Cayley graph. The elements in $E(\Gamma)$ are called the ends of $\Gamma$. Let $e(\Gamma)$ be the cardinality of $E(\Gamma)$. It can be shown that $e(\Gamma)$ is a quasi-isometric invariant of $\Gamma$. The following facts are due to Hopf and Freudenthal:

1. $e(\Gamma) \in\{0,1,2, \infty\}$.

2. $e(\Gamma)=0$ if and only if $\Gamma$ is finite.

3. $e(\Gamma)=2$ if and only if $\Gamma$ has an infinite cyclic subgroup of finite index.

It is a well-known theorem of Stalling that a finitely generated group has more than one end if and only if it is a nontrivial amalgamated free product or an HNN extension over a finite subgroup; see [3, 36, 37]. 
Lemma 3.3. Suppose we have the following short exact sequence of groups:

$$
1 \rightarrow \mathbb{Z} \rightarrow A_{1} \rightarrow A_{2} \rightarrow 1,
$$

then $A_{1}$ has one or two ends.

Proof. If $A_{2}$ is finite, $A_{1}$ contains an infinite cyclic subgroup of finite index and hence has two ends. Otherwise $A_{2}$ is infinite, it follows that $A_{1}$ has one end by Proposition 1.9 in [12].

Lemma 3.4. Suppose we have the following short exact sequence of groups:

$$
1 \rightarrow B_{1} \rightarrow B_{2} \rightarrow B_{3} \rightarrow 1,
$$

where $B_{1}$ is a cyclic group and $B_{3}$ is virtually nilpotent, then $B_{2}$ is virtually nilpotent. Here, we say a group is virtually nilpotent if it contains a nilpotent subgroup of finite index.

Proof. By passing to a subgroup of finite index, we can assume that $B_{3}$ is a nilpotent group. Since $B_{1}$ is normal in $B_{2}$, we see $B_{2}$ acts on $B_{1}$ by conjugation. This gives a group homomorphism from $B_{2}$ to $\operatorname{Aut}\left(B_{1}\right)$. Since $B_{1}$ is a cyclic $\operatorname{group}, \operatorname{Aut}\left(B_{1}\right)$ is a finite group. Hence, up to a subgroup of finite index, we get a central group extension

$$
1 \rightarrow B_{1} \rightarrow B_{2} \rightarrow B_{3} \rightarrow 1,
$$

where $B_{1}$ is cyclic and $B_{3}$ is nilpotent. Now it is easy to see $B_{2}$ is also nilpotent.

Proof of Theorem 1.4. In fact, assume $\Gamma \simeq \pi_{1}(M)$, where $M$ is a compact Sasakian manifold. If the Sasakian structure $\left(g_{0}, \xi_{0}, \eta_{0}, \phi_{0}\right)$ on $M$ is irregular, by a theorem of P. Rukimbira [32], there is a sequence of quasi-regular Sasakian structures $\left(g_{k}, \xi_{k}, \eta_{k}, \phi_{k}\right)$ converging to $\left(g_{0}, \xi_{0}, \eta_{0}, \phi_{0}\right)$ in the compact-open $C^{\infty}$ topology. So we can always assume that the Sasakian structure on $M$ is quasi-regular. Now let $M$ be an orbifold $S^{1}$ bundle over some compact Kähler orbifold $N$ and $p: M \rightarrow N$ is the projection map. Then we have the following long exact sequence

$$
\rightarrow \pi_{2}^{\text {orb }}(N) \rightarrow \mathbb{Z} \rightarrow \pi_{1}(M) \rightarrow \pi_{1}^{\text {orb }}(N) \rightarrow 1
$$

from which we get the following short exact sequence:

$$
1 \rightarrow \Gamma_{1} \rightarrow \pi_{1}(M) \rightarrow \pi_{1}^{\text {orb }}(N) \rightarrow 1,
$$

where $\Gamma_{1}$ is a cyclic group.

Now we prove the first part of Theorem 1.4. First note that $\Gamma$ cannot have two ends otherwise it contains an infinite cyclic subgroup of finite index, which is impossible by Proposition 1.1. If $\Gamma_{1}$ is an infinite cyclic group, $\Gamma$ has one end by Lemma 3.3. Otherwise $\Gamma_{1}$ is finite and $\Gamma$ is quasi-isometric to $\pi_{1}^{\text {orb }}(N)$. It follows that $\Gamma$ has zero or one end by Lemma 3.1.

Suppose $\Gamma$ is solvable, then $\pi_{1}^{\text {orb }}(N)$ is also solvable. It follows that $\pi_{1}^{\text {orb }}(N)$ is a virtually nilpotent group by Lemma 3.1 and $\Gamma$ is also virtually nilpotent by Lemma 3.4. This proves the second part of Theorem 1.4.

Proof of Theorem 1.5. First of all, compact quotients of $S^{3}$ or the three-dimensional Heisenberg group or $S \widetilde{L(2, \mathbb{R})}$ are compact Sasakian manifolds. So, one direction is easy. Now we prove the other direction. Suppose $\Gamma=\pi_{1}(M) \simeq \pi_{1}\left(V^{3}\right)$, where $M$ is a compact Sasakian manifold and $V^{3}$ is a compact three-dimensional manifold. By 
passing to the orientable cover, we can assume that $V^{3}$ is orientable. Again we can assume that $M$ is an orbifold $S^{1}$ bundle over some compact Kähler orbifold $N$ and $p: M \rightarrow N$ is the projection map. Then, we have the following long exact sequence:

$$
\rightarrow \pi_{2}^{\text {orb }}(N) \rightarrow \pi_{1}\left(S^{1}\right) \rightarrow \pi_{1}(M) \rightarrow \pi_{1}^{\text {orb }}(N) \rightarrow 1 .
$$

From which we get the following short exact sequence:

$$
1 \rightarrow i_{*}\left(\pi_{1}\left(S^{1}\right)\right) \rightarrow \pi_{1}(M) \rightarrow \pi_{1}^{\text {orb }}(N) \rightarrow 1,
$$

where $i: S^{1} \rightarrow M$ is the inclusion map. If $i_{*}\left(\pi_{1}\left(S^{1}\right)\right)=\{1\}, \Gamma=\pi_{1}(M) \simeq \pi_{1}^{\text {orb }}(N)$ and so $\Gamma$ is a Kähler orbifold group. By assumption, we have $\Gamma \simeq \pi_{1}\left(V^{3}\right)$. It is known that the fundamental groups of compact three manifolds are residually finite by the work of Perelman and Hemple; see [23, 29, 30]. Now by Lemma 3.2, $\Gamma$ contains a subgroup of finite index $\Gamma_{0}$ such that $\Gamma_{0}$ is a Kähler group. It follows that $\Gamma_{0}$ is finite by a theorem of Dimca and Suciu; see [14] and [26]. So, $\Gamma$ is finite and the universal cover of $V^{3}$ is diffeomorphic to $S^{3}$ by the work of Perelman [29, 30]. Now we assume that $i_{*}\left(\pi_{1}\left(S^{1}\right)\right)$ is a nontrivial cyclic group. Then $\pi_{1}\left(V^{3}\right)$ contains a nontrivial cyclic normal subgroup. In this case, we first

Claim. $V^{3}$ is a Seifert manifold.

Given the above claim, it follows that $V^{3}$ carries one of the geometries $S^{2} \times \mathbb{R}$, $H^{2} \times \mathbb{R}, \mathbb{R}^{3}, S^{3}, S \widetilde{L(2, \mathbb{R})}, N i l$, where $N i l$ is the three-dimensional Heisenberg group. However, those manifolds carrying one of the geometries $S^{2} \times \mathbb{R}, H^{2} \times \mathbb{R}, \mathbb{R}^{3}$ have virtually odd first Betti number. However, any Sasaki group has even first Betti number by Proposition 1.1. So $V^{3}$ has geometry modeled on $S^{3}$ or the three-dimensional Heisenberg group or $\widehat{S L(2, \mathbb{R})}$.

Proof of claim. First of all, we can assume that $V^{3}$ is prime in the sense of being indecomposable under connected sum, since a nontrivial-free product is never a Sasaki group by Theorem 1.4. By assumption, $\pi_{1}\left(V^{3}\right)$ contains a nontrivial cyclic normal subgroup $\Gamma_{0}$. Firstly, suppose $\Gamma_{0}$ is a finite cyclic group. Then $\pi_{1}\left(V^{3}\right)$ has nontrivial torsion. Since $V^{3}$ is a prime also orientable three-manifold, it follows that $\pi_{1}\left(V^{3}\right)$ must be finite by a theorem of Epstein; see [18, 22]. So, the universal cover of $V^{3}$ is diffeomorphic to $S^{3}$ by the work of Perelman. Now we assume that $\Gamma_{0}$ is an infinite cyclic group. Since $V^{3}$ is prime and orientable, it is either $S^{2} \times S^{1}$ or irreducible, that is, every embedded $S^{2}$ bounds a three-cell. Since a Sasaki group cannot be infinite cyclic by Corollary 1.3 , we see $V^{3}$ must be irreducible. On the other hand, $\pi_{1}\left(V^{3}\right)$ contains an infinite cyclic normal subgroup and so $V^{3}$ must be a Seifert manifold by a theorem of Casson, Jungreis [11] and Gabai [20].

\section{Harmonic maps from compact Sasakian manifolds}

We first state the following theorem due to Petit; see [31].

Theorem 4.1. Suppose $f$ is a harmonic map from a compact Sasakian manifold $\left(M_{1}, h_{1}\right)$ to a compact Riemannian manifold $\left(M_{2}, h_{2}\right)$ with nonpositive sectional curvature, then $f_{*}(\xi)=0$, where $\xi$ is the Reeb vector field associated to $M_{1}$. Moreover, if $M_{1}$ is a regular Sasakian manifold fibering over Kähler manifold $N$ and $p: M_{1} \rightarrow N$ is the projection map, there exists a harmonic map $g$ from $N$ to $M_{2}$ such that $f=g p$. 
It was known by Boyer and Galicki [5] that the connected sum of two compact negatively curved manifolds cannot admit Sasakian structure. The following corollary is a generalization of this result.

Corollary 4.2. There is no continuous map of nonzero degree from a compact Sasakian manifold to the connected sum $M_{1}^{n} \sharp M_{2}^{n}$, where $\left(M_{1}^{n}, g_{1}\right)$ is a compact Riemannian manifold with nonpositive sectional curvature and $M_{2}^{n}$ is any compact $n$-dimensional manifold. In particular, $M_{1}^{n} \sharp M_{2}^{n}$ cannot admit Sasakian structure.

Proof. Suppose $f_{1}$ is a continuous map of nonzero degree from a compact Sasakian manifold $\left(M_{0}^{n}, g_{0}\right)$ to $M_{1}^{n} \sharp M_{2}^{n}$. Note there is a continuous map of degree one $f_{2}$ : $M_{1}^{n} \sharp M_{2}^{n} \rightarrow M_{1}^{n}$. Let $f_{3}=f_{2} f_{1}$, then $f_{3}:\left(M_{0}^{n}, g_{0}\right) \rightarrow\left(M_{1}^{n}, g_{1}\right)$ is a continuous map of nonzero degree. By a classical theorem of Eells and Sampson [17], within the same homotopy class, we can find a harmonic map $f_{4}$. On the other hand, by Theorem 4.1, $f_{4}$ cannot be surjective by Sard's theorem. It follows that the degree of $f_{4}$ is zero. Contradiction.

As an application of Corollary 4.2, any compact manifold admitting a metric of nonpositive sectional curvature cannot admit Sasakian structure. For example, suppose $H^{3}$ is a three-dimensional hyperbolic homology sphere and $T^{2}$ is the two-dimensional torus. Then $T^{2} \times H^{3}$ cannot admit Sasakian structure by corollary 4.2. However, as far as the author knows, the nonexistence of Sasakian structure on $T^{2} \times H^{3}$ does not follow from any previously known obstructions.

Corollary 4.3. Suppose $\Gamma$ is the fundamental group of some compact regular Sasakian manifold, then $\Gamma$ cannot be a cocompact, discrete and torsion-free subgroup of $G$, where $G$ is $S O(1, n), n>2$ or $F_{4(-20)}$.

Proof. Let $M$ be a compact regular Sasakian manifold fibering over some compact Kähler manifold $N$ and $p: M \rightarrow N$ is the projection map. Let $\Gamma=\pi_{1}(M)$. We prove Corollary 4.3 by contradiction. Suppose $\Gamma \simeq \pi_{1}(B)$, where $B=\Gamma \backslash G / K$, where $K$ is a maximal compact subgroup of $G$. It follows that $B$ is a compact locally symmetry space of noncompact type and so admits a Riemannian metric of nonpositive sectional curvature. Since $B$ is an aspherical manifold, there exists a continuous map $f$ inducing the isomorphism between $\pi_{1}(M)$ and $\pi_{1}(B)$. Then by a classical result of Eells and Sampson, within the same homotopy class, there exists a harmonic map $g$. By Theorem 4.1, there exists a harmonic map $h$ from $N$ to $B$ such that $g=h p$. It follows that $p_{*}: \pi_{1}(M) \rightarrow \pi_{1}(N)$ is injective. On the other hand, from the long exact sequence of homotopy groups we know that $p_{*}$ is surjective and hence $p_{*}$ is an isomorphism. Now $\Gamma$ is a Kähler group and also a cocompact lattice in $G$, where $G$ is $S O(1, n), n>2$ or $F_{4(-20)}$. This is impossible by the results of Carlson, Hernández and Toledo; see $[8,9]$.

\section{A transversal Jacobi equation and proof of Theorem 1.6}

The proof of Theorem 1.6 is based on the following transversal Cartan-Hadamard theorem:

Theorem 5.1. Suppose $\left(M^{2 n+1}, g\right)$ is a complete Sasakian manifold with nonpositive transversal sectional curvature, then its universal cover is diffeomorphic to $\mathbb{R}^{2 n+1}$. 
Corollary 5.2. Suppose $\left(M^{2 n+1}, g\right)$ is a compact Sasakian manifold with nonpositive transversal sectional curvature, then its fundamental group cannot be Gromov hyperbolic.

In fact, by a theorem of Mineyev [27, 28], any compact aspherical manifold has positive simplicial volume if its fundamental group is Gromov hyperbolic. Since any compact Sasakian manifold has vanishing simplicial volume, Corollary 5.2 follows.

By the classification of compact Sasakian manifolds in dimension 3 ([5, 21]), we get

Corollary 5.3. Suppose $\left(M^{3}, g\right)$ is a three-dimensional compact Sasakian manifold with nonpositive transversal sectional curvature, then $M^{3}$ is diffeomorphic to the compact quotient of the three-dimensional Heisenberg group or $\widetilde{S(2, \mathbb{R})}$.

On the other hand, compact quotients of the three-dimensional Heisenberg group $(\widehat{S L(2, \mathbb{R}))}$ are Seifert circle bundles over flat (hyperbolic) orbifolds and so admit Sasakian structure with nonpositive transversal sectional curvature.

Before we prove Theorem 5.1, we show how to derive Theorem 1.6 from it. We prove it by contradiction. Suppose $\Gamma=\pi_{1}(M, g) \simeq \pi_{1}(N, h)$, where $(M, g)$ is a compact Sasakian manifold with nonpositive transversal sectional curvature and $(N, h)$ is a compact Riemannian manifold with nonpositive sectional curvature. Since $N$ is an aspherical manifold, there exists a continuous map $f$ inducing the isomorphism between fundamental groups. Then by a classical result of Eells and Sampson, within the same homotopy class, there exists a harmonic map $g$. By a theorem of Banyaga and Rukimbira $([2,33,34])$, there exists a closed leaf of the characteristic foliation on $M$. Since $M$ is compact, we see that this leaf is diffeomorphic to $S^{1}$. From the proof of Theorem 5.1, we see that this $S^{1}$ is lifted to a real line under the covering map $p: \widetilde{M} \rightarrow M$, where $\widetilde{M}$ is the universal cover of $M$. This implies that the inclusion map $i_{*}: \pi_{1}\left(S^{1}\right) \rightarrow \pi_{1}(M)$ is injective. On the other hand, since $g$ is harmonic, $g_{*}(\xi)=0$ by Theorem 4.1, where $\xi$ is the Reeb vector field associated to $M$. So $g_{*}$ maps a nontrivial subgroup of $\Gamma$ to zero, which contradicts that $g_{*}$ is an isomorphism.

Now we are in position to prove Theorem 5.1. The idea is similar to the proof of the Cartan-Hadamard theorem. Suppose $\widetilde{M^{2 n+1}}$ is the universal cover of $M^{2 n+1}$. Then it is also a complete Sasakian manifold with nonpositive transversal sectional curvature. Suppose $\alpha(s)$ is a leaf of the characteristic foliation $F_{\xi}$ on $\widetilde{M^{2 n+1}}$. We show that its normal exponential map $\exp ^{\perp}: \alpha(s)^{\perp} \rightarrow \widetilde{M^{2 n+1}}$ is a diffeomorphism, where $\alpha(s)^{\perp}$ is the normal bundle of $\alpha(s)$. To do this, suppose $\gamma(t), t \in[0,1]$ is any minimizing geodesic which is perpendicular to $\alpha(s)$ at $\alpha(0)=\gamma(0)$ and $J(t)$ is any Jacobi field along $\gamma(t)$ such that $J(0)=\lambda \xi(0)$ and $J(1)=0$, where $\xi(0)=\alpha^{\prime}(0)$. It suffices to show $J(t) \equiv 0$. Then we see that there is no focal point to $\alpha(s)$. It follows that $\exp ^{\perp}$ is a covering map and hence is a diffeomorphism since $\widetilde{M^{2 n+1}}$ is simply connected.

There is a natural splitting $T_{\gamma(t)} M=\xi(t) \oplus \xi(t)^{\perp}$, where $\xi(t)$ is the tangential part to leaves of the characteristic foliation $F_{\xi}$ and $\xi(t)^{\perp}$ is the orthogonal part. Following Wilking [42], define $Y(t)=J^{\perp}(t)$ and $\nabla_{\frac{\partial}{\partial t}}^{\perp} Y=\left(\nabla_{\frac{\partial}{\partial t}} Y\right)^{\perp}=\left(\nabla_{\dot{\gamma}} Y\right)^{\perp}$, where we denote $X^{\perp}(t)$ is the projection of $X(t)$ to $\xi(t)^{\perp}$ for any vector field $X(t)$ along $\gamma(t)$. 
Lemma 5.4. $Y(t)$ satisfies the following transversal Jacobi equation:

$$
\nabla \frac{\partial}{\partial t} \nabla_{\frac{\partial}{\partial t}}^{\perp} Y+R^{T}(Y, \dot{\gamma}) \dot{\gamma}=0
$$

Before we prove it, we need the following lemma.

\section{Lemma 5.5.}

$$
R^{T}(X, Y) Y=(R(X, Y) Y)^{\perp}+3\langle X, \phi(Y)\rangle \phi(Y), \quad \text { where } X, Y \perp \xi .
$$

Proof. Choose any vector field $Z$ such that $Z \perp \xi$. We see

$$
\begin{aligned}
R^{T}(X, Y, Y, Z)= & \left\langle R^{T}(X, Y) Y, Z\right\rangle=\left\langle\nabla_{X}^{T} \nabla_{Y}^{T} Y-\nabla_{Y}^{T} \nabla_{X}^{T} Y-\nabla_{[X, Y]}^{T} Y, Z\right\rangle \\
= & \left\langle\nabla_{X}\left(\nabla_{Y} Y-\left\langle\nabla_{Y} Y, \xi\right\rangle \xi\right)-\nabla_{Y}\left(\nabla_{X} Y-\left\langle\nabla_{X} Y, \xi\right\rangle \xi\right)\right. \\
& \left.-\nabla_{[X, Y]-\langle[X, Y], \xi\rangle \xi}^{T} Y-\nabla_{\langle[X, Y], \xi\rangle \xi}^{T} Y, Z\right\rangle \\
= & \langle R(X, Y) Y, Z\rangle+\left\langle\nabla_{X} Y, \xi\right\rangle\left\langle\nabla_{Y} \xi, Z\right\rangle+\langle[X, Y], \xi\rangle\left\langle\nabla_{Y} \xi, Z\right\rangle \\
= & \langle R(X, Y) Y, Z\rangle+3\langle X, \phi(Y)\rangle\langle\phi(Y), Z\rangle .
\end{aligned}
$$

where the last equality follows from $X, Y \perp \xi$ and $\xi$ is a Killing vector field.

Now, we are in position to prove Lemma 5.4. It suffices to prove it at generic $t_{0}$, i.e., $J\left(t_{0}\right) \neq 0$. Firstly note that we can assume that $Y\left(t_{0}\right)=J\left(t_{0}\right)$. Choose vector fields $X_{i}(t), i=1,2, \ldots, 2 n$ such that $X_{i} \perp \xi$ and also $J\left(t_{0}\right)=X_{1}\left(t_{0}\right), \nabla_{\frac{\partial}{\partial t}}^{\perp} X_{i}(t)=$ $0,\left\langle X_{i}, X_{j}\right\rangle(t)=\delta_{i j}$ for all $t \in[0,1]$. Note that we have

$$
X_{i}^{\prime}=\left\langle X_{i}^{\prime}, \xi\right\rangle \xi=\left\langle\nabla_{\dot{\gamma}} X_{i}, \xi\right\rangle \xi=-\left\langle X_{i}, \nabla_{\dot{\gamma}} \xi\right\rangle \xi
$$

using this, at $t_{0}$, we have

$$
\begin{aligned}
\left\langle\nabla_{\frac{\partial}{\partial t}}^{\perp} \nabla_{\frac{\partial}{\partial t}}^{\perp} Y, X_{i}\right\rangle= & \left\langle\nabla_{\frac{\partial}{\partial t}} \nabla_{\frac{\partial}{\partial t}}^{\perp} Y, X_{i}\right\rangle \\
= & \frac{\partial}{\partial t}\left\langle\nabla_{\frac{\partial}{\partial t}}^{\perp} Y, X_{i}\right\rangle-\left\langle\nabla_{\frac{\partial}{\partial t}}^{\perp} Y, \nabla_{\frac{\partial}{\partial t}}^{\perp} X_{i}\right\rangle \\
= & \frac{\partial}{\partial t}\left\langle\nabla_{\frac{\partial}{\partial t}}^{\perp} Y, X_{i}\right\rangle=\frac{\partial^{2}}{\partial t^{2}}\left\langle J, X_{i}\right\rangle \\
= & \left\langle J^{\prime \prime}, X_{i}\right\rangle+2\left\langle J^{\prime}, X_{i}^{\prime}\right\rangle+\left\langle J, X_{i}^{\prime \prime}\right\rangle \\
= & -\left\langle R(Y, \dot{\gamma}) \dot{\gamma}, X_{i}\right\rangle+2\left\langle J^{\prime}, X_{i}^{\prime}\right\rangle+\left\langle X_{1}, X_{i}^{\prime \prime}\right\rangle \\
= & -\left\langle R(Y, \dot{\gamma}) \dot{\gamma}, X_{i}\right\rangle+2\left\langle J^{\prime}, X_{i}^{\prime}\right\rangle \\
& +\frac{d}{d t}{ }_{t=t_{0}}\left\langle X_{1}, X_{i}^{\prime}\right\rangle-\left\langle X_{1}^{\prime}, X_{i}^{\prime}\right\rangle \\
= & -\left\langle R(Y, \dot{\gamma}) \dot{\gamma}, X_{i}\right\rangle+2\left\langle J^{\prime}, X_{i}^{\prime}\right\rangle-\left\langle X_{1}^{\prime}, X_{i}^{\prime}\right\rangle \\
= & -\left\langle R(Y, \dot{\gamma}) \dot{\gamma}, X_{i}\right\rangle-2\left\langle J^{\prime}, \xi\right\rangle\left\langle X_{i}, \xi^{\prime}\right\rangle-\left\langle X_{1}, \xi^{\prime}\right\rangle\left\langle X_{i}, \xi^{\prime}\right\rangle \\
= & -\left\langle R(Y, \dot{\gamma}) \dot{\gamma}, X_{i}\right\rangle-2\left\langle J, \xi^{\prime}\right\rangle\left\langle X_{i}, \xi^{\prime}\right\rangle-\left\langle J, \xi^{\prime}\right\rangle\left\langle X_{i}, \xi^{\prime}\right\rangle \\
= & \left\langle-R^{T}(Y, \dot{\gamma}) \dot{\gamma}, X_{i}\right\rangle,
\end{aligned}
$$

where the last equality follows from Lemma 5.5 and the last second equality follows from

\section{Lemma 5.6.}

$$
\left\langle J^{\prime}(t), \xi(t)\right\rangle=\left\langle J(t), \xi^{\prime}(t)\right\rangle
$$


Proof.

$$
\begin{aligned}
\left\langle J^{\prime}(t), \xi(t)\right\rangle & =\left\langle\nabla_{\dot{\gamma}} J, \xi\right\rangle(t)=\left\langle\nabla_{J} \dot{\gamma}, \xi\right\rangle(t)=-\left\langle\dot{\gamma}, \nabla_{J} \xi\right\rangle(t) \\
& =\left\langle J, \nabla_{\dot{\gamma}} \xi\right\rangle(t)=\left\langle J(t), \xi^{\prime}(t)\right\rangle .
\end{aligned}
$$

Now define $f(t)=\frac{1}{2}\|Y(t)\|^{2}$, then $f(0)=f(1)=0$, since $Y(0)=Y(1)=0$. Moreover, we have

$$
f^{\prime}(t)=\left\langle\nabla_{\frac{\partial}{\partial t}}^{\frac{1}{\partial t}} Y, Y\right\rangle
$$

and also

$$
\begin{aligned}
f^{\prime \prime}(t) & =\left\langle\nabla_{\frac{\partial}{\partial t}}^{\perp} Y, \nabla_{\frac{\partial}{\partial t}}^{\perp} Y\right\rangle+\left\langle\nabla_{\frac{\partial}{\partial t}}^{\perp} \nabla_{\frac{\partial}{\partial t}}^{\perp} Y, Y\right\rangle \\
& =\left\langle\nabla_{\frac{\partial}{\partial t}}^{\perp} Y, \nabla_{\frac{\partial}{\partial t}}^{\perp} Y\right\rangle-R^{T}(Y, \dot{\gamma}, \dot{\gamma}, Y) \geq 0,
\end{aligned}
$$

from this we know $f(t)$ is a convex nonnegative function with $f(0)=f(1)=0$ and so $f(t)=0$ for any $t \in[0,1]$. So $Y(t) \equiv 0$ and we can assume that $J(t)=\lambda(t) \xi(t)$ with $\lambda(0)=\lambda$. Then $\lambda(t)=\langle J(t), \xi(t)\rangle$ and $\lambda(1)=0$ since $J(1)=0$. Taking derivative with respect to $t$, we get

$$
\begin{aligned}
\lambda^{\prime}(t) & =\left\langle J^{\prime}(t), \xi(t)\right\rangle+\left\langle J(t), \xi^{\prime}(t)\right\rangle \\
& =2\left\langle J(t), \xi^{\prime}(t)\right\rangle=2\left\langle\lambda(t) \xi(t), \xi^{\prime}(t)\right\rangle=0 .
\end{aligned}
$$

so $J(t) \equiv 0$ and $\exp ^{\perp}: \alpha(s)^{\perp} \rightarrow \widetilde{M^{2 n+1}}$ is a diffeomorphism. Hence, $\widetilde{M^{2 n+1}}$ is diffeomorphic to the normal bundle of a one-dimensional manifold. Since $\widetilde{M^{2 n+1}}$ is simply connected, we see $\widehat{M^{2 n+1}}$ is diffeomorphic to $\mathbb{R}^{2 n+1}$.

\section{Abel-Jacobi maps of compact Sasakian manifolds}

We first recall the construction of the Abel-Jacobi map of any compact Riemannian manifold; see [24] for more details.

Suppose $(M, g)$ is a compact Riemannian manifold. Let $\pi=\pi_{1}(M)$ be its fundamental group. Let $f: \pi \rightarrow \pi^{a b}$ be the abelianization map of $\pi$ and $g: \pi^{a b} \rightarrow \pi^{a b} /$ tor be the quotient by torsion. Suppose $\bar{M}$ is the covering space of $M$ with $\pi_{1}(\bar{M})=\operatorname{ker}(\phi)$, where $\phi=g f$.

Let $E$ be the space of harmonic one-form on $M$, with dual $E^{*}$ canonically identified with $H_{1}(M, \mathbb{R})$. Fix a basepoint $x_{0} \in M$. Then any point $x$ in the universal cover $\tilde{M}$ of $M$ is represented by a point of $M$ together with a path $c$ from $x_{0}$ to it. By integrating along the path $c$, we get a linear form, $h \rightarrow \int_{c} h$, on $E$. We thus obtain a map $\tilde{M} \rightarrow E^{*}=H_{1}(M, \mathbb{R})$, which descends to a map

$$
\overline{A_{M}}: \bar{M} \rightarrow E^{*}, c \mapsto\left(h \mapsto \int_{c} h\right) .
$$

By definition, the Jacobi torus of $M$ is the torus

$$
J_{1}(M)=H_{1}(M, \mathbb{R}) / H_{1}(M, \mathbb{Z})_{\mathbb{R}}
$$


and the Abel-Jacobi map

$$
A_{M}: M \rightarrow J_{1}(M),
$$

is obtained from the map $\overline{A_{M}}$ by passing to quotients. From the construction, it is not hard to see the Abel-Jacobi map induces an isomorphism between the first homology groups with real coefficient.

Proposition 6.1. Suppose $(M, g)$ is a compact Sasakian manifold and $F_{\xi}$ is the characteristic foliation on $M$. Then the restriction of the Abel-Jacobi map of $M$ to any leaf of $F_{\xi}$ is a constant map.

Proof. By a theorem of Tachibana [38], any harmonic one form $h$ on $M$ satisfies $h(\xi)=0$. Using it, Proposition 6.1 easily follows from the construction of the Abel-Jacobi map.

Corollary 6.2. Suppose $(M, g)$ is a compact Sasakian manifold and $i: S^{1} \rightarrow M$ is the inclusion map, where $S^{1}$ is a closed leaf of the characteristic foliation $F_{\xi}$ on $M$. Then $i_{*}\left(\pi_{1}\left(S^{1}\right)\right) \subseteq \operatorname{ker} \phi$, where $\phi$ is the map constructed in the beginning of this section. In other words, $S^{1}$ generates a trivial element in $H_{1}(M, \mathbb{R})$.

Proof. By Proposition 6.1, the Abel-Jacobi map of $M$ takes $S^{1}$ to a point. However, the Abel-Jacobi map induces an isomorphism between the first homology groups with real coefficient and so $S^{1}$ generates a trivial element in $H_{1}(M, \mathbb{R})$.

We end this paper by proposing several problems to be studied in the future.

Problem 1: How large is the class of Sasaki groups which are not Kähler groups?

Problem 2: Classify those nilpotent groups which are also Sasaki groups;

Problem 3: Are there examples of irregular compact Sasakian manifolds with nonpositive transversal sectional curvature?

\section{Acknowledgments}

The author would like to express his gratitude to his thesis advisor, Professor Karsten Grove, for insistent encouragement and helpful advice. He also thanks Professor Charles Boyer, Marc Burger, Frederic Campana, Qintao Deng, Gaofeng Zheng for many helpful discussions.

\section{References}

[1] J. Amorós, M. Burger, K. Corlette, D. Kotschick and D. Toledo, Fundamental groups of compact Kähler manifolds, in 'Mahtematical Surveys and Monographs', 44 American Mathematical Society, Providence, RI, 1996.

[2] A. Banyaga, A note on Weinstein's conjecture, Proc. Amer. Math. Soc. 109(3) (1990), 855-858.

[3] G. Bergman, On groups acting on locally finite graphs, Ann. Math. 88 (1968), 335-340.

[4] D. Blair and S. Goldberg, Topology of almost contact manifolds, J. Differ. Geom. 1 (1967), $347-354$.

[5] C. Boyer and K. Galicki, Sasakian geometry, Oxford University Press, Oxford, 2008.

[6] F. Campana, Orbifoldes gomtriques spciales et classification bimromorphe des varits khlriennes compactes, J. Inst. Math. Jussieu 10(4) (2011), 809-934. 
[7] — Quotients résoluble ou nilpotents des groupes de Kähler orbifoldes, Manus. Math. 135(1-2) (2011), 117-150.

[8] J. Carlson and L. Hernández, Harmonic maps from compact Kähler manifolds to exceptional hyperbolic spaces, J. Geom. Anal. 1(4) (1991), 339-357.

[9] J. Carlson and D. Toledo, Harmonic mappings of Kähler manifolds to locally symmetric spaces, Publ. Math. L'IHÉS 69(1) (1989), 173-201.

[10] - Quadratic presentations and nilpotent Kähler groups, J. Geom. Anal. 5(3) (1995), 359-377.

[11] A. Casson and D. Jungreis, Convergence groups and Seifert fibered 3-manifolds, Invent. Math. 118(1) (1994), 441-456.

[12] D. Cohen, Ends and free products of groups, Math. Z. 114(1) (1970), 9-18.

[13] M.W. Davis, Lectures on orbifolds and reflection groups, Hangzhou, China, 2008.

[14] A. Dimca and A. Suciu, Which 3-manifold groups are Kähler groups, J. Eur. Math. Soc. 11 (2009), 521-528.

[15] C. Drutu and M. Kapovich, Lectures on geometric group theory, http://www.math.ucdavis. edu/ kapovich/EPR/ggt.pdf

[16] P. Eberlein, A canonical form for compact nonpositively curved manifolds whose fundamental groups have nontrivial center, Math. Ann. 260(1) (1982), 23-29.

[17] J. Eells and J. Sampson, Harmonic mappings of Riemannian manifolds, Amer. J. Math. 86(1) (1964), 109-160.

[18] D. Epstein, Projective planes in 3-manifolds, Proc. London. Math. Soc. s3-11 (1961), 469-484.

[19] T. Fujitani, Complex-valued differential forms on normal contact Riemannian manifolds, Tôhoku Math. J. 18(4) (1966), 349-361.

[20] D. Gabai, Convergence groups are Fuchsian groups, Ann. Math 136(2) (1992), 447-510.

[21] H. Geiges, Normal contact structures on 3-manifolds, Tôhoku Math. J. 49(3) (1997), 415-422.

[22] J. Hempel, 3-Manifolds, Ann. Math. Stud. 86, American Mathematical Society, 1976.

[23] — Residual finiteness for 3-manifolds, Combinatorial group theory and topology 111 (1987), 379-396.

[24] M. Katz, Systolic geometry and topology, Mathematical Surveys and Monographs, 137 (2007).

[25] S. Kobayashi, Topology of positively pinched Kähler manifolds, Tôhoku Math. J. 15(2) (1963), 121-139.

[26] D. Kotschick, Three-manifolds and Kähler groups, 2011, arXiv:1011.4084v3

[27] I. Mineyev, Straightening and bounded cohomology of hyperbolic groups, Geom. Funct. Anal. 11(4) (2001), 807-839.

[28] — Bounded cohomology characterizes hyperbolic groups, Q. J. Math. 53(1) (2002), 59-73.

[29] G. Perelman, The entropy formula for the Ricci flow and its geometric applications, 2002, arXiv:0211159.

[30] — Ricci flow with surgery on three-manifolds, 2003, arXiv:0303109.

[31] R. Petit, Harmonic maps and strictly pseudoconvex CR manifolds, Comm. Anal. Geom. 10(3) (2002), 575-610.

[32] P. Rukimbira, Chern-Hamilton's conjecture and K-contactess, Houston. J. Math 21(4) (1995), 709-718.

[33] - Topology and closed characteristics of K-contact manifolds, Bull. Belgian Math. Soc. Simon Stevin 2(3) (1995), 349-356.

[34] - On K-contact manifolds with minimal number of closed characteristics, Proc. Amer. Math. Soc. 127 (1999) 3345-3351.

[35] C. Simpson, Local systems on proper algebraic V-manifolds, 2010, arXiv:1010.3363.

[36] J. Stallings, On torsion-free groups with infinitely many ends, Ann. Math. 88(2) (1968), 312-334.

[37] - Group theory and three-dimensional manifolds, 83, Yale University Press, New Haven, 1971.

[38] S. Tachibana, On harmonic tensors in compact Sasakian spaces, Tôhoku Math. J. 17(3) (1965), $271-284$.

[39] S. Tanno, Harmonic forms and Betti numbers of certain contact Riemannian manifolds, J. Math. Soc. Japan 19(3) (1967), 308-316. 
[40] W. Thurston and J. Milnor, The geometry and topology of three-manifolds, Princeton University Lecture Notes (1978-1981).

[41] A. Wadsley, Geodesic foliations by circles, J. Differ. Geom. 10(4) (1975), 541-549.

[42] B. Wilking, A duality theorem for Riemannian foliations in nonnegative sectional curvature, Geom. Funct. Anal. 17(4) (2007), 1297-1320.

Department of Mathematics, University of Notre Dame, Notre Dame, IN 46556, USA

E-mail address: xchen3@nd.edu 
